Journal of Animal and Veterinary Advances 11 (16): 2969-2975, 2012

ISSN: $1680-5593$

(C) Medwell Journals, 2012

\title{
Cannabinoid Receptor Type 1 (CB1) Antagonist, SR141716 Suppresses Hepatic CPT1 Gene Expression in Rat
}

\author{
Ting Wu, Xiufeng Yu, Linna Zhu and Yizhen Wang \\ Institute of Feed Science, Zhejiang University, No. 388 Yuhangtang Road, \\ Hangzhou, 310058 Hangzhou, P.R. China
}

\begin{abstract}
SR141716 is the antagonist of Cannabinoid Receptor type 1 (CB1). The study was conducted to investigate the effects of SR141716 on body weight gain, body fat, hepatic fat deposit and Carnitine Palmitoyltransferase 1 (CPT1) expression in obese Sprague-Dawley (SD) rats induced by High Fat Diet (HFD). Twenty four SD rats were randomly allocated to three groups: the Normal Diet (ND) as control group, the High Fat Diet (HFD) and SR141716 treated HFD-diet (SFD) animals as experimental groups. Plasma was collected immediately for following plasma indexes determination. Liver samples were flash frozen in lipid nitrogen and stored at $-80^{\circ} \mathrm{C}$ until use for following experiments. Compared with the ND group, HFD significantly increased the body weight gain $(\mathrm{p}<0.05)$, total viscera fat pad $(\mathrm{p}<0.05)$ and hepatic Triglyceride $(\mathrm{TG})(\mathrm{p}<0.05)$ in rats while SR141716 significantly suppressed these effects $(\mathrm{p}<0.05)$. Furthermore, hepatic CPT1 mRNA level was significant decreased by HFD $(\mathrm{p}<0.05)$. Hepatic CPT1 and PPAR $\gamma$ mRNA level were significantly suppressed by SR141716 accompany with CB1 mRNA expression decrease $(\mathrm{p}<0.05)$. The results indicated that SR141716 can significantly suppress the excess hepatic adipose deposit induced by HFD in rats. During this process, the pivotal role of CB1 in hepatic fat deposit may considerably to a large extent be due to modulation of CPT1 expression through PPAR $\gamma$. However, the accurate mechanism remains to be elucidated in the future.
\end{abstract}

Key words: SR141716, antagonist, visceral fat deposition, hepatic fat deposition, body weight, CB1, CPT1, mRNA level, high fat diet, rat

\section{INTRODUCTION}

Fat storage and adipose tissue distribution in animals have gained increasing attention in developed countries as well as developing countries. Almost all animal species dispose the residual energy through storing as fat pad in adipose tissues or some other organs until their biological energy needs (Van Vleet et al., 1984; McKay et al., 2003). When energy intake excesses expenditure, obesity will develop. Now a days, being overweight or obese and its associated disease such as fatty liver, type 2 diabetes, dyslipidemias, cardiovascular disease, Alzheimer's disease, gallstones and even some cancers are developing a significant global health threat to human beings (Nakamura et al., 1994). Obesity, especially with visceral and hepatic fat accumulation is a serious risk factor for the so-called metabolic syndrome. The increase of hepatic diseases epidemic draw more and more studies pointing to the investigation of pivotal interaction between genes that could be helpful for predispose to obesity. Liver as a significant site of lipid and glucose oxidation, accounts for as much as $30 \%$ of basal energy expenditure
(Zurlo et al., 1990) and especially functions as a pivotal adipose metabolism and insulin resistant in mammals (Osei-Hyiaman et al., 2008). Most of mortal diseases are of great part due to dysfunction of hepatic energy metabolism not only in animals but also in human beings.

Several recent studies in obese humans and animals suggest that SR141716 have significant anti-obesity effects through suppressing hepatic fat deposit (Doyon et al., 2006). SR141716 is a specific antagonist of $\mathrm{CB} 1$, also named rimonabant, the first discovered selective CB1 antagonist (Rinaldi-Carmona et al., 1994) which exhibits as a promise anti-obesity reagent when administered to diet-induced obese mice and rats (Ravinet et al., 2003; Doyon et al, 2006). Van Gaal et al. (2005) and Despres et al. (2005) report that SR141716 not only reduced body weight but also abated cardiovascular risk associated with obesity. However, the precise mechanism responsible for the suppression of SR141716 on hepatic fat accumulation remains to be elucidated. Osei-Hyiaman et al. (2005) has established that activation of CB1 in wild type mice stimulates de novo lipogenesis in the liver through induction of the lipogenic

Corresponding Author: Yizhen Wang, Institute of Feed Science, Zhejiang University, No. 388 Yuhangtang Road, Hangzhou, 310058 Hangzhou, P.R. China 
transcription factor SREBP1 and its target enzymes Acetyl-CoA Carboxylase (ACC1) and Fatty Acid Synthase (FAS). Furthermore, it has been reported that peripheral CB1 could be selectively targeted for the treatment of fatty liver while high-fat diet feeding failed to reduce CPT1 activity in either $\mathrm{CB} 1-/$ - or LCB1-/- mice but CPT1 mRNA levels were markedly reduced in wild type mice fed with high fat diet versus normal diet (Osei-Hyiaman et al., 2008). Although, these findings strongly implicate hepatic CB1 in the development of diet-induced obesity, related metabolic changes and indirect relationship between CB1 and the rate limiting enzyme in fatty acid $\beta$-oxidation (CPT1) was investigated but direct knowledge about precise mechanism of CB1 influencing hepatic fat accumulation through CPT1 remains to be eagerly supplemented.

Therefore, this study was conducted to evaluate the efficacy of SR141716 in suppressing visceral and hepatic fat accumulation and influencing lipolysis-related markers (CPT1) in a rat with HFD-induced obesity in vivo and to provide knowledge for elucidating the molecular mechanisms responsible for this effects in the future.

\section{MATERIALS AND METHODS}

Animals: All experimental male Sprague-Dawley (SD) rats, aged 4-5 weeks were purchased from Experimental Animal Centre in Zhejiang University (Hangzhou, China). All rats were cared for and handled according to the guide and rules of care and use of laboratory animals. The experimental animals were acclimated to their new experimental environment for 7 days prior to be allocated to experiment setup during which time they were allowed free access to a standard pellet diet according to the standard experimental diet (GB 14924-2001, China) which composed of $10.4 \%$ water, $5.8 \%$ crude ash, $24.5 \%$ crude protein, $8.53 \%$ crude fat, $47.1 \%$ nitrogen-free extract. The gross energy of the standard diet was calculated to be $3932 \mathrm{cal} \mathrm{g}^{-1}$ chow.

Experiment design: Twenty four experimental SD rats were randomly allocated to three groups with 8 individuals of equal body weights in each group: the normal diet as control group (CND, 8\% fat), the High Fat Diet (HFD with 35\% fat) and HFD-diet treated with SR141716 (99.2\% in NMT, Shanghai Me-too Pharma Tech Co., Ltd. China) animals groups as experimental studies. All rats were housed in wire-bottom cages in a room where the temperature was kept at $21 \pm 2^{\circ} \mathrm{C}$, the relative humidity at $50 \pm 5 \%$ and the light at a $12 \mathrm{~h}$ light/dark cycle (lights on between 0800 and 2000) and were allowed unrestricted access to water. Treated rats received a daily oral administration of $10 \mathrm{mg} \mathrm{kg}^{-1} \mathrm{SR} 141716$ for 21 days at 0830 except on the last day where SR141716 was administrated $6 \mathrm{~h}$ before death. This dose was earlier reported to reduce bodyweight in fat rats (Bensaid et al., 2003; Vickers et al., 2003). SR141716 was given orally in a a solution with $2 \mathrm{mg} \mathrm{mL}^{-1} \mathrm{SR} 141716$ and $2 \mu \mathrm{L} \mathrm{mL}^{-1}$ Tween-80 which have been previously reported by Osei-Hyiaman et al. (2005) and was administered orally by gavage in a volume of $5 \mathrm{~mL} \mathrm{~kg}{ }^{-1} \mathrm{BW}$. Rats were weighed daily at 0830 throughout the experiment. Rats were killed between 1400 and 1700 after 8-12 h deprivation. All the rats were anesthetized with ether and then were killed by cervical dislocation. Liver, muscle from hindlimb and longissium dorsi muscle were sampled immediately after cervical dislocation. The perirenal, epididymal and mesenteric adipose tissues were removed and weighed as total viscera fat pad. Samples were flash frozen in liquid nitrogen and stored at $-80^{\circ} \mathrm{C}$ for RNA extraction and following experiments.

Detection of hepatic triglyceride deposit: Liver samples were freshly collected immediately after cervical dislocation for detecting triglyceride deposition. Approximately $0.2-1.0 \mathrm{~g}$ liver tissues from each sample were rinsed with $0.86 \%$ icephysiological saline for removing the blood and infranatant adhere to the tissues. Weigh the net fresh sample and then minced the liver samples with $9 \mathrm{w} / \mathrm{v}$ of $0.86 \%$ icephysiological saline on ice. Supernatants were collected as $10 \%$ minced liver tissue solution for following hepatic triglyceride deposition determination by centrifuging the minced tissues at $2,500 \mathrm{rpm}$, for $15 \mathrm{~min}$ at $4^{\circ} \mathrm{C}$. Triglyceride deposit in liver was assayed by kit according to the commercial manufacturer's instructions (Biotech Limit Corp. Jiancheng, Nanjing, China).

Plasma determination: Blood was collected from inferior vena cava into sterile tube and the serum was obtained by centrifuging the blood at $1,500 \mathrm{~g}$ for $15 \mathrm{~min}$ at $4^{\circ} \mathrm{C}$. Plasma was stored at $-20^{\circ} \mathrm{C}$ until later biochemical measurements. Plasma total cholesterol concentrations were determined using an automated glucose analyzer YSI 2,300 Stat Plus (YSI, Yellow Springs, $\mathrm{OH}$ ). Commercially available radioimmunoassay kits (Linco Research, St. Charles, MO) were used to determine plasma levels of free fatty acids whereas enzymatic kits were used for triglycerides (Roche Diagnostics, Laval, Quebec, Canada).

RNA extraction: Total RNA was extracted from collected tissues by Trizol reagent (Invitrogen) according to the manufacture's protocol. RNA quality was assessed by OD260nm calculation method and all the ratios of 
OD260/OD280nm were between 1.8 and 2.0 . Approximately $1 \mu \mathrm{g}$ total RNA was used for reverse transcription using thermoscript RT-PCR system (Invitrogen) and random primers and the specific procedure was carried out according to methods of Shan et al. (2008). The cDNA products were firstly ensured by $18 \mathrm{sec}$ rRNA amplifying PCR before Real-Time PCR detection.

Real-time quantitative RT-PCR: Real-time PCR was performed using SYBR green nucleic acid dye on an applied Biosystems 7500 Fast Real-Time PCR System (Applied Biosystems, Foster City, CA) to quantify CB1, $C P T 1$ and PPAR $\gamma$ gene expression level. The real time RTPCR primer pairs were shown in Table 1. Primer pairs of PPAR y (F: 5'-tgg gtg aaa ctc tgg gag at -3 ', R: 5'-cca tag tgg aag cet gat gc $-3^{\prime}$ ) and L27 (F: 5'-ctg ctc get gtc gaa atg $-3^{\prime}, \mathrm{R}: 5^{\prime}$-cct tge gtt tca gtg ctg $-3^{\prime}$ ) was designed according to published papers and have been confirmed before use (Pang et al., 2008; Festuccia et al., 2006). Data are expressed as the ratio between the expression of the target gene and the housekeeping gene L27 which was selected because no significant variation in its expression was observed between control and treated rats. L27 mRNA expression level was used as internal control in all real-time PCR detection, following this thermal profile: $94^{\circ} \mathrm{C} 2 \mathrm{~min}$ followed by 40 cycles of $95^{\circ} \mathrm{C} 30 \mathrm{sec}, 60^{\circ} \mathrm{C}$ $45 \mathrm{sec}$. To calculate the expression of mRNA expression $C B 1, C P T 1$ and PPAR $\gamma$ gene, the CT values was used for detection of CB1, CPT1 and PPAR $\gamma$ mRNA related to internal control $L 27$ gene expression using the $2-\Delta \Delta C T$ Method (Livak and Schmittgen, 2001; Shan et al., 2008). The PCR reaction contained $1 \mu \mathrm{L}$ diluted cDNA in a $20 \mu \mathrm{L}$ $\mathrm{PCR}$ reaction. These three target genes were normalized to the housekeeping gene, L27 mRNA levels and displayed as a relative fold-change compared to those of ND rats. The dissociation/melting curves yielded single peaks were presented here indicating all the products with no contamination or lack of primer dimmers.

Statistical analysis: Statistical analysis was carried out through ANOVA and all data are presented here as means \pm SEM. A $\mathrm{p}<0.05$ displaying here indicates statistically significance.

Table 1: Primers sequences for $C B 1, C P T 1$ gene in rat

\begin{tabular}{llcc}
\hline Genes & Primer sequences & Length (bp) & AccessionNo. \\
\hline$C B 1$ & F: 5'-caa gca cgc caa caa cac a -3' & & \\
& R: 5'-tct taa cgg tgc tct tga tgc a -3' & 68 & NM012784 \\
$C P T 1$ & F: 5'-cag aac acg gca aaa tga gc -3' & & \\
& R: 5'-gag gtt gac agc aaa atc ctg-3' & 122 & NM013200 \\
\hline
\end{tabular}

$\mathrm{F}=$ Sense primer, $\mathrm{R}=$ Antisense primer

\section{RESULTS AND DISCUSSION}

Body weight gain and visceral fat-pad weight: $R$ at treated with the HFD attained $173.8 \pm 2.58 \mathrm{~g}$ of cumulative body weight gain in 20 days, exhibiting significantly more body weight gain by $9.62 \%$ compared with that of rats fed with ND ( $p<0.05)$. SR141716 inverse this influence, notably reducing body weight gain by 11.01 and $2.41 \%$ compared with rats fed with HFD $(\mathrm{p}<0.05)$ and ND $(\mathrm{p}<0.05)$, respectively (Fig. 1). The relative weights of the visceral fat deposit were much higher in the HFD-rats than the ND-rats showed in Fig. 1. $(\mathrm{p}<0.05)$. However, the SR141716 significantly diminished the effects of high fat diets on visceral fat pad of experimental rats $(\mathrm{p}<0.05)$.

Plasma triglyceride, total cholesterol, free fatty acids assays: The plasma triglyceride, total cholesterol and fatty acids levels were detected using commercial reagent kits and the data were shown in Fig. 2. The plasma triglyceride and fatty acids were exhibited higher level in rats fed with HFD compared with those of ND-rats $(p<0.05)$ while SR141716 remarkably reduce triglyceride and free fatty acids enrichment in blood $(\mathrm{p}<0.05)$. These phenomena appeared without significantly altering the total cholesterol level in the treated animals ( $>0.05$ ).

Triglyceride deposit in liver: The results of Triglyceride deposit in liver were showed in Fig. 3. Triglyceride contents in liver of experimental rats were elevated significantly due to treatment of HFD $(p<0.01)$. With the increase of fat in diet but feeding SR141716 synchronously, rats' triglyceride deposition in liver showed dramatically suppressed $(\mathrm{p}<0.05)$.

Hepatic gene expression profile in experimental animals: High fat and SR141716 mix-induced gene expression profile was evaluated using real time PCR analyses (Fig. 4). In this study, CB1, CPT1 and PPAR $\gamma$ gene mRNA level were determined as relative to housekeeping gene $L 27$ mRNA level which would not be variable during treating with HFD or SR141716. The mRNA level of $C B 1$ and CPT1 genes in liver tissues of HFD-rats were decreased by $24.89 \%(p<0.05)$ and $24.85 \%(p<0.05)$, respectively compared with those of ND-rats. Simultaneously, PPAR $\gamma$ gene mRNA level was significantly elevated by $26.69 \%(\mathrm{p}<0.05)$ when contrasted to those of rats fed with ND. Moreover, SR141716 further boost the decrease of $C B 1$ and CPT1 genes mRNA levels obviously $(\mathrm{p}<0.05)$ compared with those of ND-rats or HFD-rats. However, surprisingly, SR141716 conducts no enhancement of PPAR $\gamma$ gene mRNA level but notably inverse the excessive gain of gene mRNA level induced by HFD $(\mathrm{p}<0.05)$. 

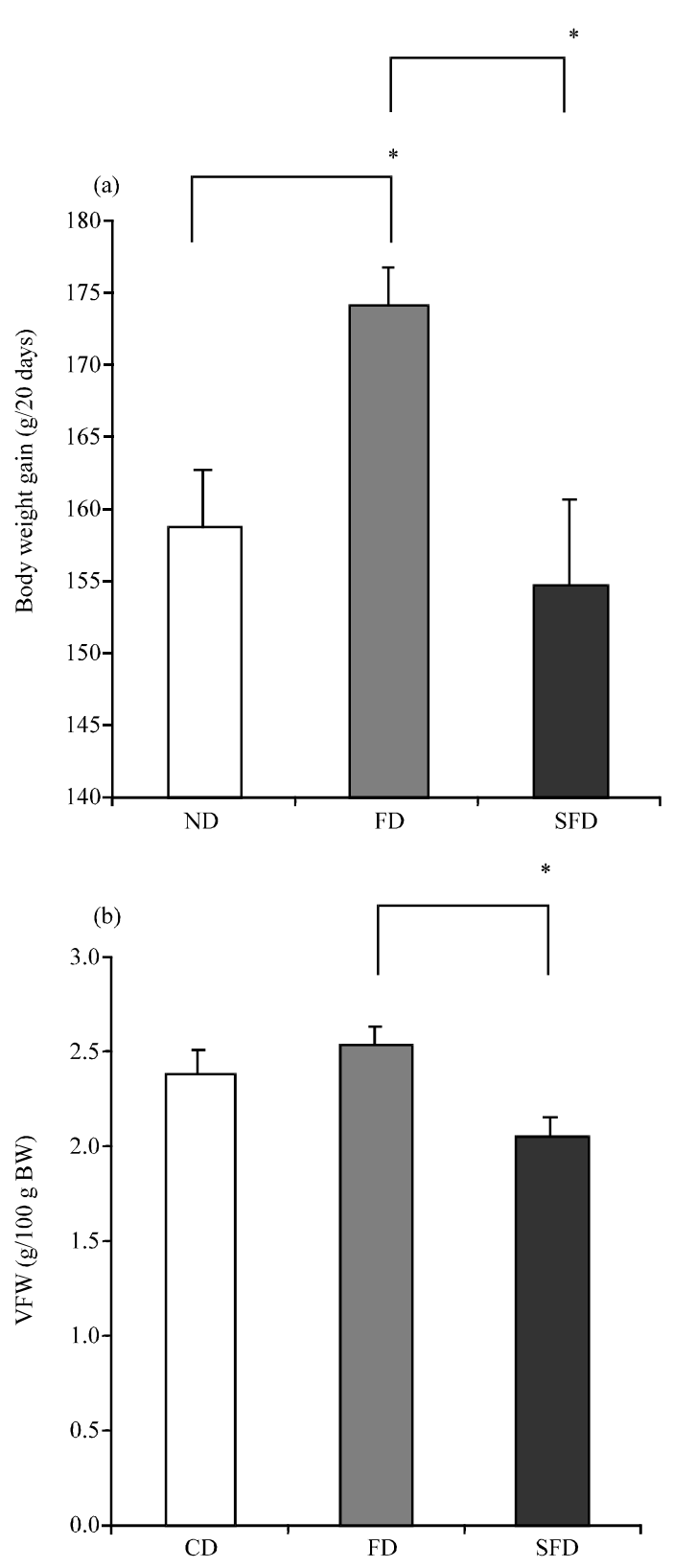

Fig. 1: a) Body weight gain of rats fed with Normal Diet (ND) as control, High Fat Diet (HFD) and high fat diet supplemented with administrating SR141716 (SFD) for 20 experimental days; b) Visceral fat pad weight of rats fed with $\mathrm{ND}, \mathrm{HFD}$ and SFD for whole experimental period. Values presented as mean \pm SEM, $n=8 .{ }^{*} \mathrm{p}<0.05$

It has been reported that $<5 \%$ of TG is synthesized in liver and adipose tissue themselves. Most Free Fatty Acids (FFAs) stored as TG in adipose tissues and liver are derived from diet (Wang et al., 2008). Therefore, agents administered orally that could regulate food intake and energy usage may be the first important step for the

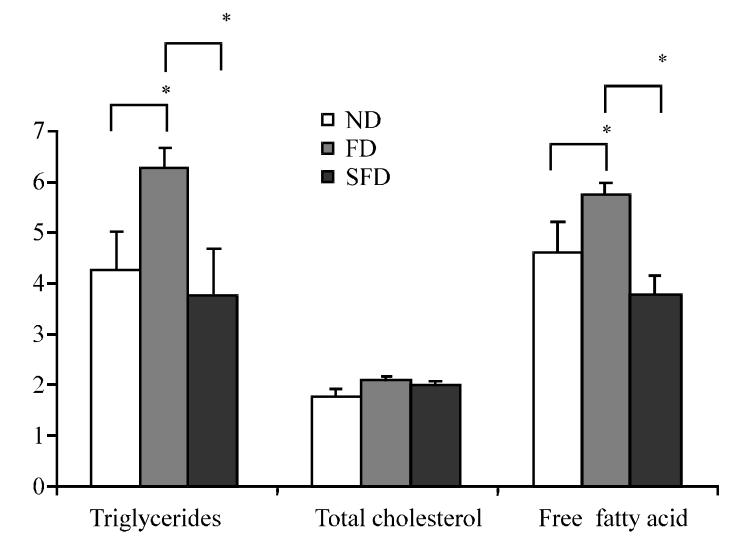

Fig. 2: Plasma triglyceride ( $\mathrm{mmol} / 10 \mathrm{~L})$, total cholesterol $\left(\mathrm{mmol} \mathrm{L} \mathrm{L}^{-1}\right)$, free fatty acids $\left(100 \mu \mathrm{mol} \mathrm{L} \mathrm{L}^{-}\right)$ assays of rats fed with ND, HFD and SFD for whole experimental period. Values presented as mean \pm SEM, $n=8 .{ }^{*} \mathrm{p}<0.05$

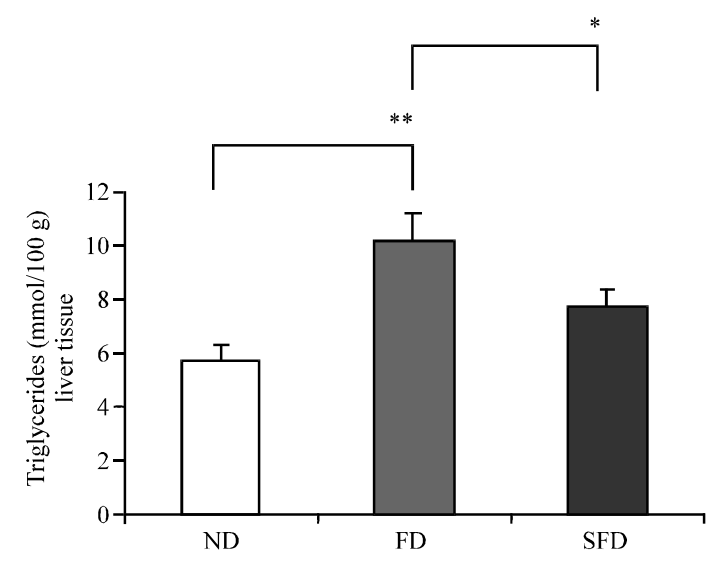

Fig. 3: Triglyceride deposit determination in liver of rats fed with ND, HFD and SFD for whole experimental period. Values presented as mean \pm SEM, $\mathrm{n}=8$. ${ }^{*} \mathrm{p}<0.05,{ }^{* *} \mathrm{p}<0.01$

program of anti-obesity and clinical therapies. To the forth, large amount of earlier published results have demonstrated that antagonist or agonist of CB1, AM4113, rimonabant (SR141716) and AM251 are of great extent selectively for CB1, respectively (McLaughlin et al., 2006; Sink et al., 2008, 2009). A number of recent observations suggest that the suppression or activation of $\mathrm{CB} 1$ using agonist or inverse agonist is a central factor in regulation of appetite and diet induced obesity (Doyon et al., 2006; Osei-Hyiaman et al., 2008, 2005; Sink et al., 2009). In the current study, researchers use SR141716 to directly treat the high fat diet induced rats accompanying with complete visceral fat and hepatic fat deposit analysis. Results showed that SR141716 suppressing the 

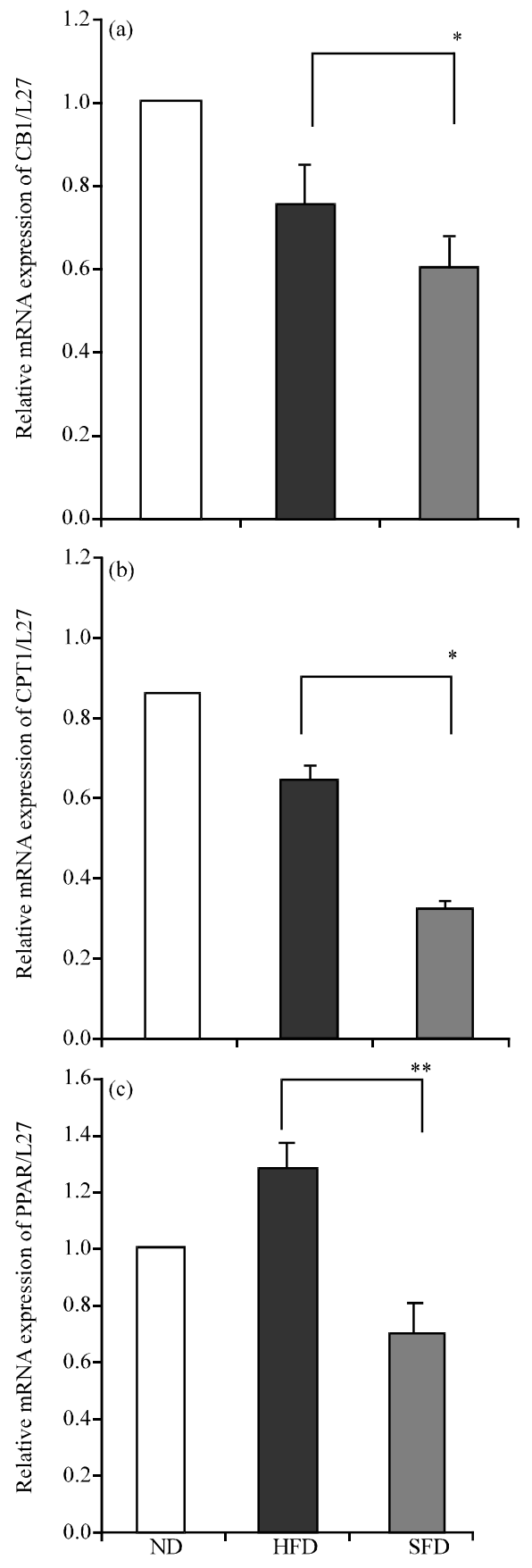

Fig. 4: a) CB1, b) CPT1 and c) PPAR y mRNA levels determined by real time RT-PCR analyses in liver tissues of rats fed with ND, HFD and SFD for whole experimental period. Results were normalized to the housekeeping gene $L 27 \mathrm{mRNA}$ levels. The CB1, CPT1 and PPAR $\gamma$ mRNA levels of rats fed with HFD and SFD were exhibited as the fold change contrast to ND rats. Values are means \pm SEM of 8 duplicated analyses of RNA samples from 8 rats. ${ }^{*} \mathrm{p}<0.05,{ }^{*} \mathrm{p}<0.01$ experimental rat bodyweight gain was largely account for the reduction in fat gain including visceral fat gain which is consistence to the previous reports (Vickers et al., 2003; Gomez et al., 2002; Freedland et al., 2003).

To further explain the impact of SR141716 on fat metabolism in liver and investigate the possible molecular regulative mechanisms, researchers assessed its influence on hepatic TG and CB1 mRNA levels using quantitative real time RT-PCR. It has been reported recently that antagonist of CB1 could significantly decrease the visceral fat pad deposition of rats induced by SR141716 administration. This study further boosts up previous conclusion. With the increasing concern about the disease related to obesity such as liver disease, growing evidence indicates that CB1 antagonists not only have potent anti-inflammatory activity but also have a beneficial effect on liver disease such as liver fibrosis, obesity-associated hepatic steatosis and alcoholic fatty liver (Gary-Bobo et al., 2007; Jeong et al., 2008; Teixeira-Clerc et al., 2006). Kojima et al. (2009) raise the possibility of using CB1 antagonists as anti-inflammatory drugs for treating hepatitis as well as other inflammatory diseases. Ragan et al. (2009) report that antagonist of CB1, CP-945,598-01 is an efficient drug for the treatment of obesity. This study demonstrated that SR141716 not only exhibits important role in suppression in body fat deposit but also prevent excess TG stored in liver when rats exposed to high fat diet. SR141716 as a selective and very affinitive agent of CB1, exactly attenuated the hepatic CB1 expression in rats treated with HFD which greatly augmented the reports that SR141716 could selectively suppress CB1 expression previously studied in vivo and in vitro (Guo et al., 2009; Serrano et al., 2008; Gary-Bobo et al., 2007; Tedesco et al., 2008).

Furthermore, based on the Osei-Hyisman's research group's recent report that hepatic CPT1 expression level was not affected by high fat diet when CB1 was blocked while versus results were detected in wild type mice. Researchers hypothesize that SR141716 may attenuate the suppression effect of HFD on CPT1. It is very consist with the hypothesis that CPT1 mRNA level was reduced in liver due to HFD treatment. However, to the surprise, the SR141716 did not attenuate but augment the suppression effect of HFD on CPT1 mRNA level. This may be explained in part by the recent published results that display a complex signaling interaction between CB1 and other receptors such as dopamine D2 and Orexinl receptors. It is also worthwhile to present Hilairet's reports that SR141716 may prevent weight gain through functional interaction between CB1 and other receptors involved in the control of appetite which may probably be exist in liver. This results may also partly due to the 
suppression of SR141716 on plasma TG and FFA, abating the supply of TGs and FFA from blood which attenuate the lipid synthesis and lipolysis process because most FFAs stored as TG in adipose tissues and liver are derived from diet mentioned previously (Wang et al., 2008).

Therefore, basing on the present results, PPAR $y$ as a key transcriptional factor, its expression in liver was also detected during this experiment. Seal obviously develop that PPAR $y$ exhibits indispensable role in the process of PRDM1 6 regulating energy balance to influence animal's obesity and fat distribution. Therefore, PPAR $\gamma$ was determined in the study, results showed that SR141716 significantly suppressed the enhancement of PPARy expression induced by HFD. From these results, researchers assumably deduce that PPAR $y$ may exhibit important regulating function in the process of CB1 and CPT1 interaction. However, whether PPAR $\gamma$ plays indispensable role in CB1 regulating of CPT1 program remains unclear. More and more hepatic fat deposit associated genes were still needed detailed investigation for elucidating the precise mechanisms of CB1 regulating CPT1 signaling pathways.

\section{CONCLUSION}

This study have clearly demonstrated that SR141716 can significantly reduce the body, visceral and hepatic TG deposit of obese rats induced by HFD without negative effects. Moreover, researchers find that SR141716 apparently ameliorate the liver fat accumulation of HFD treated rats. These effects are probably mediated by CB1 regulation through suppressing lipid metabolism in liver which may be accounted for its suppression of CPT1 mRNA expression. Furthermore, PPAR $\gamma$ may also play pivotal role in the process of CB1 regulating CPT1 expression during hepatic fat accumulation.

\section{ACKNOWLEDGEMENTS}

This research was supported by the National Basic Research Program of China (2009ZX08009-144B). The researchers thank Jia Guo, Yang Ren for their kindly help in the experiments.

\section{REFERENCES}

Bensaid, M., M. Gary-Bobo, A. Esclangon, J.P. Maffrand, G. Le Fur, F. Oury-Donat and P. Soubrie, 2003. The cannabinoid CB1 receptor antagonist SR141716 increases Acrp30 mRNA expression in adipose tissue of obese fa/fa rats and in cultured adipocyte cells. Mol. Pharmacol., 63: 908-914.
Despres, J.P., A. Golay and L. Sjostrom, 2005. Effects of rimonabant on metabolic risk factors in overweight patients with dyslipidemia. New Engl. J. Med., 353: 2121-2134.

Doyon, C., R.G. Denis, E.D. Baraboi, P. Samson, J. Lalonde, Y. Deshaies and D. Richard, 2006. Effects of rimonnabant (SR141716) on fastinginduced hypothalamic pituitary adrenal axis and neuronal activation in lean and obese zucker rats. Diabetes, 55: 3403-3410.

Festuccia, W.T., M. Laplante, M. Berthiaume, Y. Gelinas and Y. Deshaies, 2006. PPAR $y$ agonism increases rat adipose tissue lipolysis, expression of glyceride lipases and the response of lipolysis to hormonal control. Diabetologia, 49: 2427-2436.

Freedland, C.S., C.T. Whitlow, H.R. Smith and L.J. Porrino, 2003. Functional consequences of the acute administration of the cannabinoid receptor antagonist, SR141716A, in cannabinoid-naive andtolerant animals: A quantitative 2-[14C] deoxyglucose study. Brain Res., 962: 169-179.

Gary-Bobo, M., G. Elachouri, J.F. Gallas, P. Janiak and P. Marini et al., 2007. Rimonabant reduces obesityassociated hepatic steatosis and features of metabolic syndrome in obese zucker fa/fa rats. Hepatologym, 46: 122-129.

Gomez, R., M. Navarrol, B. Ferrer, J.M. Trigo and A. Bilbao et al., 2002. A peripheral mechanism for CB1 cannabinoid receptor-dependent modulation of feeding. J. Neurosci., 22: 9612-9617.

Guo, Y., G. Wu, X. Su, H. Yang and J. Zhang, 2009. Antiobesity action of a daidzein derivative on male obese mice induced by a high-fat diet. Nutr. Res., 29: 656-663.

Jeong, W.I., D. Osei-Hyiaman, O. Park, J. Liu and S. Batkai et al., 2008. Paracrine activation of hepatic CB1 receptors by stellate cell-derived endocannabinoids mediates alcoholic fatty liver. Cell Metab., 7: 227-235.

Kojima, M., N. Kato, D. Hirano, T. Ochi, A. Nii, K. Shinjo and H. Eda, 2009. Selective CB1 cannabinoid receptor antagonist, SR141716A, attenuates liver injury induced by concanavalin A. Hepatol Res., 39: 408-414.

Livak, K.J. and T.D. Schmittgen, 2001. Analysis of relative gene expression data using Real-time quantitative PCR and the 2 (delta delta $\mathrm{C} \mathrm{(} \mathrm{T} \mathrm{)} \mathrm{method.} \mathrm{Methods,}$ 25: 402-408.

McKay, R.M., J.P. McKay, L. Avery and J.M. Graff, 2003. C. elegans: A model for exploring the genetics of fat storage. Dev. Cell, 4: 131-142. 
McLaughlin, P.J., L. Qian, J.T. Wood, A. Wisniecki and K.M. Winston et al., 2006. Suppression of food intake and food-reinforced behavior produced by the novel CB1 receptor antagonist/inverse agonist AM 1387. Pharmacol. Biochem. Behav., 83: 396-402.

Nakamura, T., K. Tokunaga, I. Shimomura, M. Nishida and S. Yoshida et al., 1994. Contribution of visceral fat accumulation to the development of coronary artery disease in non-obese men. Atherosclerosis, 107: 239-246.

Osei-Hyiaman, D., J. Liu, L. Zhou, G. Grezgorz and J. Harvey-White et al., 2008. Hepatic CB1 receptor is required for development of diet-induced steatosis dyslipidemia and insulin and leptin resistance in mice. J. Clin. Invest., 118: 3160-3169.

Osei-Hyiaman, D., M. DePetrillo, P. Pacher, J. Liu and S. Radaeva et al., 2005. Endocannabinoid activation at hepatic CB1 receptors stimulates fatty acid synthesis and contributes to diet-induced obesity. J. Clin. Invest, 115: 1298-1305.

Pang, J., Y. Choi and T. Park, 2008. Ilex paraguariensis extract ameliorates obesity induced by high-fat diet: Potential role of AMPK in the visceral adipose tissue. Arch. Biochem. Biophys., 476: 178-185.

Ragan, J.A., D.E. Bourassa, J. Blunt, D. Breen and F.R. Busch et al., 2009. Development of a practical and efficient synthesis of CP-945,598-01, a CB1 antagonist for the treatment of obesity. Org. Process Res. Dev., 13: 186-197.

Ravinet, T.C., M. Arnone, C. Delgorge, N. Gonalons, P. Keane, J.P. Maffrand and P. Soubrie, 2003. Anti-obesity effect of SR141716, a CB1 receptor antagonist, in diet-induced obese mice. Am. J. Physiol. Regul. Integr. Comp. Physiol., 284: 345-353.

Rinaldi-Carmona, M., F. Barth, M. Heaulme, D. Shire and B. Calandra et al., 1994. SR141716A, a potent and selective antagonist of the brain cannabinoid receptor. FEBS Lett., 350: 240-244.

Serrano, A., I. Del-Arco, F. Javier-Pavon, M. Macias, V. Perez-Valero and F. Rodriguez-de-Fonseca, 2008. The cannabinoid CB1 receptor antagonist SR141716A (Rimonabant) enhances the metabolic benefits of long-term treatment with oleoylethanolamide in zucker rats. Neuropharmacol., 54: $226-234$.
Shan, T., Y. Wang, T. Wu, J. Guo, J. Liu, J. Feng and $\mathrm{Z}$. $\mathrm{Xu}, 2008$. Porcine adipose triglyceride lipase complementary deoxyribomucleic acid clone, expression pattern and regulation by resveratrol. J. Anim. Sci., 86: 1781-1788.

Sink, K.S., P.J. McLaughlin, J.A. Wood, C. Brown and P. Fan et al., 2008. The novel cannabinoid CB1 receptor neutral antagonist AM4113 suppresses food intake and food-reinforced behavior but does not induce signs of nausea in rats. Neuropsychopharmacol., 33: 946-955.

Sink, K.S., V.K. Vemuri, J. Wood, A. Makriyanni and J.D. Salamone, 2009. Oral bioavailability of the novel cannabinoid CB1 antagonist AM6527: Effects on food-reinforced behavior and comparisons with AM41 13. Pharmacol. Biochem. Behav., 91: 303-306.

Tedesco, L., A. Valerio, C. Cervino, A. Cardile and C. Pagano et al., 2008. Cannabinoid Type 1 receptor blockade promotes mitochondrial biogenesis through endothelial nitric oxide synthase expression in white adipocytes. Diabetes, 57: 2028-2036.

Teixeira-Clerc, F., B. Julien, P. Grenard, J.T.V. Nhieu and V. Deveaux et al., 2006. CB1 cannabinoid antagonism: A new strategy for the treatment of liver fibrosis. Nat. Med., 12: 671-676.

Van Gaal, L.F., A.M. Rissanen, A.J. Scheen, O. Ziegler and S. Rossner, 2005. Effects of the cannabinoid-1 receptor blocker rimonabant on weight reduction and cardiovascular risk factors in overweight patients: 1 year experience from the RIO-Europe study. Lancet, 365: 1389-1397.

Van Vleet, E.S., S. Candileri, J. McNeillie, S.B. Reinhardt, M.E. Conkright and A. Zwissler, 1984. Neutral lipid components of eleven species of caribbean sharks. Comp. Biochem. Physiol., 79: 549-554.

Vickers, S.P., L.J. Webster, A. Wyatt, C.T. Dourish and G.A. Kennett, 2003. Preferential effects of the cannabinoid CB1 receptor antagonist, SR 141716, on food intake and body weight gain of obese $(\mathrm{fa} / \mathrm{fa}$ ) compared to lean Zucker rats. Psychopharmacol., 167: 103-111.

Wang, S., K.G. Soni, M. Semache, S. Casavant, M. Fortier, L. Pan and G.A. Mitchell, 2008. Lipolysis and the integrated physiology of lipid energy metabolism. Mol. Genet Metab., 95: 117-126.

Zurlo, F., K. Larson, C. Bogardus and E. Ravussin, 1990. Skeletal muscle metabolism is a major determinant of resting energy expenditure. J. Clin. Invest., 86: $1423-1427$. 